\title{
The Group Inverse of the Nivellateur
}

\author{
R.E. Hartwig* P. Patrício ${ }^{\dagger}$
}

\begin{abstract}
We shall derive necessary and sufficient conditions for the Nivellateur to have a group inverse over an algebraically closed field. We then extend these results to arbitrary fields.
\end{abstract}

Keywords: Nivellateur, group inverse, matrices over a field

AMS classification: 15A09, 15A21

\section{The nivellateur}

The matrix equation $A X-X B=C$ can be written in column form as $G \operatorname{vec}(\mathrm{X})=\operatorname{vec}(\mathrm{C})$, where $\operatorname{vec}(Y)=\left[\begin{array}{c}\mathbf{y}_{1} \\ \vdots \\ \mathbf{y}_{n}\end{array}\right]$ when $Y=\left[\begin{array}{lll}\mathbf{y}_{1} & \cdots & \mathbf{y}_{n}\end{array}\right]$, and

$$
G=I \otimes A-B^{T} \otimes I
$$

is the nivellateur of $A$ and $B$.

*Mathematics Department, N.C.S.U., Raleigh, NC 27695-8205, U.S.A. e-mail: hartwig@unity.ncsu.edu

${ }^{\dagger}$ CMAT - Centro de Matemática and Departamento de Matemática e Aplicações, Universidade do Minho, 4710-057 Braga, Portugal. e-mail: pedro@math.uminho.pt

Partially supported by FCT-'Fundação para a Ciência e a Tecnologia', within the project UID-MAT00013/2013.

Corresponding author. 
Our aim is to find necessary and sufficient conditions for the existence of the group inverse of this matrix in terms of $A$ and $B$, and to provide expressions for this group inverse.

We shall use $r(X), \nu(X), R(X), R S(X), N(X)$ to denote rank, nullity, range, rowspace, nullspace of $X$, respectively.

Throughout let $A$ be $m \times m$ and $B$ be $n \times n$.

A matrix $A$ has a group inverse if there exists a solution to the equations

$$
A X A=A, \quad X A X=X, A X=X A,
$$

in which case the solution is unique and is denoted by $A^{\#}$. We shall refer to this existence as " $A$ is GP".

We begin with the easiest case, which is that of a closed field.

\section{The closed field Case}

Consider the matrices $A$ and $B$ over a closed field $\mathbb{F}$, with characteristic polynomials

$$
\Delta_{A}(x)=|x I-A|=\prod_{k=1}^{s(A)}\left(x-\lambda_{k}\right)^{n_{k}(A)}=\prod_{i=1}^{n(A)}\left(x-\alpha_{i}\right)
$$

and

$$
\Delta_{B}(x)=|x I-B|=\prod_{k=1}^{s(B)}\left(x-\mu_{k}\right)^{n_{k}(B)}=\prod_{i=1}^{n(B)}\left(x-\beta_{i}\right) .
$$

Here the $\lambda_{k}, \mu_{r}$ are distinct and the $\alpha_{i}, \beta_{i}$ may be repeated. Further let $\sigma(A)=\left\{\lambda_{1}, . ., \lambda_{s}\right\}$ be the spectrum of distinct eigenvalues of $A$ and let $\tau(A)=\left(\alpha_{1}, \ldots, \alpha_{m}\right)$ be the list of all of its $m$ eigenvalues - repeated or not. Set $T=\sigma(A) \cap \sigma(B)$.

We denote the algebraic and geometric multiplicities of $\lambda_{k}(A)$ by $n_{k}(A)$ and $\nu_{k}(A)=$ $\operatorname{dim}\left[N\left(A-\alpha_{k} I\right)\right]$ respectively.

It is clear that $\sum_{k=1}^{s(A)} n_{k}(A)=n(A)=m$ and $\sum_{j=1}^{s(B)} n_{j}(B)=n(B)=n$.

Furthermore, suppose that the minimal polynomial of $A$ is given by

$$
\psi_{A}(x)=\prod_{k=1}^{s}\left(x-\lambda_{k}\right)^{m_{k}(A)}
$$

with $m_{i}(A) \leq n_{i}(A)$. We shall refer to the exponent $m_{k}(A)$ as the index $\operatorname{ind}\left(\lambda_{k}\right)$ of $\lambda_{k}$.

It is well known that the group inverse exists if and only if the geometric and algebraic multiplicities of the zero eigenvalue are equal. 
We shall compute the algebraic multiplicity $n_{0}(G)$ and the geometric multiplicity $\nu_{0}(G)$ of the zero eigenvalue of $G$.

From Stephanos' theorem (see [6, Theorem 1, page 411]) we know that the eigenvalues of $G$ have the form $\lambda_{i j}(G)=\lambda_{i}(A)-\lambda_{j}(B)$ with $i=1, \ldots, m$ and $j=1, \ldots, n$, counted according to multiplicity. This immediately tell us that

$$
n_{0}(G)=\sum_{\gamma \in \sigma(A) \cap \sigma(B)} n_{\gamma}(A) n_{\gamma}(B) .
$$

To get more information about $G$, we first reduce $B$ to its Jordan form, via

$$
Q^{-1} B Q=J_{B}=\operatorname{diag}\left(J_{q_{1}}\left(\beta_{1}\right), . ., J_{q_{u}}\left(\beta_{u}\right)\right)
$$

where $J_{k}(a)=\left[\begin{array}{cccc}a & 1 & & 0 \\ 0 & a & 1 & \\ & & & \\ & \ddots & \ddots & 1 \\ & & & a\end{array}\right]$ and $Q$ is a suitable invertible matrix, made up of

Jordan Chains of generalized e-vectors. The $\beta_{j}$ may be repeated and $u$ is the number of Jordan blocks. The associated elementary divisors of $B$ are given by

$$
\mathcal{E}_{B}=\left\{\left(x-\beta_{j}\right)^{q_{j}} ; j=1, \ldots, u\right\}
$$

Likewise the elementary divisors of $A$ are given by $\mathcal{E}_{A}=\left\{\left(x-\alpha_{i}\right)^{p_{i}} ; i=1, \ldots, t\right\}$.

Transforming $G$ we have

$$
\left(Q^{T} \otimes I\right) G\left[\left(Q^{T}\right)^{-1} \otimes I\right]=I \otimes A-J_{B}^{T} \otimes I=\operatorname{diag}\left(G_{1}, . ., G_{s}\right)
$$

where

$$
G_{i}=I \otimes A-J_{q_{i}}^{T}\left(\beta_{i}\right)=\left[\begin{array}{cccc}
A-\beta_{i} I & & & 0 \\
-I & A-\beta_{i} I & \\
& \ddots & \ddots & \\
0 & & -I & A-\beta_{i} I
\end{array}\right]_{\text {of block size } q_{i} \times q_{i}}
$$

which will also give (2).

We now observe that if $A \mathbf{u}=\mathbf{0}$ and $B^{T} \mathbf{v}=\mathbf{0}$ then $G(\mathbf{v} \otimes \mathbf{u})=\mathbf{0}$. This means that

$$
N\left(B^{T}\right) \otimes N(A) \subseteq N(G)
$$


and hence on taking dimensions

$$
\nu(A) \cdot \nu(B) \leq \nu(G)
$$

Consequently we have (product rule)

$$
\nu(G)=\nu(A) \cdot \nu(B) \Leftrightarrow N(G)=N\left(B^{T}\right) \otimes N(A) .
$$

Let us now refine the block form of (3) to obtain:

(i) an expression for $\nu(G)$ in terms of $A$ and $B$,

(ii) conditions for $G$ to have a group inverse, and

(iii) give a formula for $G^{\#}$.

We shall then use the expression for $\nu(G)$ to show when precisely the product rule holds and when $\nu(G)=n_{0}(G)$, i.e. when $G^{\#}$ exists.

We begin with

Lemma 2.1. Let $R$ be a ring with unity 1 , and suppose that

$$
J_{n}(-a)=\left[\begin{array}{cccc}
a & & & 0 \\
-1 & a & & \\
& \ddots & \ddots & \\
0 & & -1 & a
\end{array}\right] \text { and } K_{n}(a)=\left[\begin{array}{ccccc}
1 & & & & 0 \\
a & 1 & & \\
a^{2} & \ddots & \ddots & \\
\vdots & & & \\
a^{n-1} & & & a & 1
\end{array}\right]
$$

are over $R$ with $n \geq 2$. Then

(i) $K_{n}(a)^{T} J_{n}(-a)=\left[\begin{array}{cc}0 & a^{n} \\ I & \mathbf{b}\end{array}\right]$, where $\mathbf{b}^{T}=\left[a^{n-1}, . . a^{2}, a\right]$.

(ii) $J_{n}(-a)^{\#}$ exists iff $a^{-1}$ exists. In which case $J_{n}(-a)^{\#}=J_{n}(-a)^{-1}=\left[\begin{array}{cccc}a^{-1} & & & 0 \\ a^{-2} & a^{-1} & 0 & \\ \vdots & & \ddots & \\ a^{-n} & \ldots & & \\ & & & a^{-1}\end{array}\right]$.

\section{Proof. (i) Clear.}

(ii) Equating $(2,1)$ entries in $J_{n}(-a)^{2} X=J_{n}(-a)$ and $(\mathrm{n}, \mathrm{n}-1)$ entries in $Y J_{n}(-a)^{2}=$ $J_{n}(-a)$ we see that a has both left and right inverses. 
From (3) we know that $G^{\#}$ exists iff each of the blocks $G_{i}$ has a group inverse. Now when $\beta_{i}$ is not an eigenvalue of $\mathrm{A}$ then $G_{i}$ is invertible and there is no contribution to $\nu(G)$. So we only need to consider a common eigenvalue $\gamma=\alpha_{i}=\beta_{j}$.

So let $\gamma \in T=\sigma(A) \cap \sigma(B)$ and assume that the associated elementary divisors are

$$
\mathcal{E}_{A}=\left\{(x-\gamma)^{p_{1}(\gamma)}, \ldots,(x-\gamma)^{p_{k}(\gamma)}\right\}
$$

and

$$
\mathcal{E}_{B}=\left\{(x-\gamma)^{q_{1}(\gamma)}, \ldots,(x-\gamma)^{q_{t}(\gamma)}\right\}
$$

respectively, where $p_{1}(\gamma) \geq p_{2}(\gamma) \geq \cdots \geq p_{k}(\gamma) \geq 1$ and $q_{1}(\gamma) \geq q_{2}(\gamma) \geq \cdots \geq q_{t}(\gamma) \geq 1$.

There are two cases that can happen.

(i) If $q_{i}>1$ then by Lemma 2.1 we know that $G_{i}^{\#}$ exists iff $(A-\gamma I)^{-1}$ exists, that is, iff $\gamma \notin \sigma(A)$. So this case cannot occur.

(ii) If $q_{i}=1$, i.e when we have a linear elementary divisor $x-\gamma$ in $\mathcal{E}_{B}$, then $G_{i}^{\#}$ exists iff $(A-\gamma I)^{\#}$ exists. This happens exactly when $\gamma$ is a simple root of $\psi_{A}(x)$.

Thus,

Theorem 2.1. $G^{\#}$ exists if and only if for every $\gamma \in \sigma(A) \cap \sigma(B)$ with $q_{i}=1$ (a $1 \times 1$ Jordan block) we have $\operatorname{ind}_{A}(\gamma)=1$.

In other words, for a common eigenvalue all associated elementary divisors for $A$ and $B$ must be linear.

As a by-product we can compute the nullity of $G$ [5]. Indeed, suppose that $A$ is in Jordan form, say $A=A_{\gamma} \oplus X$, where $A_{\gamma}=\operatorname{diag}\left(J_{p_{1}}(\gamma), \ldots, J_{p_{r}}(\gamma)\right)$, and $X$ contains Jordan blocks with non common eigenvalues. Note that $\nu\left(A_{\gamma}\right)=r$. Then $I \otimes A_{\gamma}-J_{q_{j}}(\gamma) \otimes I$ takes the form

$$
G_{i, j}=\left[\begin{array}{cccc}
J_{p_{1}}(0) & & & 0 \\
-I & J_{p_{2}}(0) & & \\
& \ddots & \ddots & \\
0 & & -I & J_{p_{r}}(0)
\end{array}\right]_{q_{j} \text { blocks }}
$$

Now because $\nu\left[J_{n}(0)\right]^{k}=\min (n, k)$ we see that

$$
\nu\left(G_{i j}\right)=\sum_{i=1}^{r} \min \left\{p_{i}, q_{j}\right\}
$$

Repeating this for all common eigenvalues we arrive at, c.f. [5], 


$$
\nu(G)=\sum_{\gamma \in T} \sum_{j=1}^{r} \sum_{i=1}^{r} \min \left\{p_{i}, q_{j}\right\} .
$$

Let us now use this result to derive a couple of special cases.

If $T=\emptyset$, there are no common eigenvalues and $\nu(G)=0$. In particular $0 \notin T$ and either $A$ or $B$ is invertible. Hence $\nu(A) \cdot \nu(B)=0$ and the product rule holds.

If there are common eigenvalues, but 0 is not one of them, then $\nu(A) \cdot \nu(B)=0<\nu(G)$.

Lastly, if 0 is a common eigenvalue, then separating off the common zero eigenvalue we get

$$
\nu(G)=\sum_{i=1}^{\nu(A)} \sum_{j=1}^{\nu(B)} \min \left\{p_{i}(0), q_{j}(0)\right\}+\sum_{0 \neq \alpha \in T} \sum_{i=1} \sum_{j=1} \min \left\{p_{i}(\alpha), q_{j}(\alpha)\right\} \geq \nu(A) \cdot \nu(B) .
$$

This we rewrite as

$$
\nu(G)-\nu(A) \nu(B)=\sum_{i=1}^{\nu(A)} \sum_{j=1}^{\nu(B)}\left[\min \left\{p_{i}(0), q_{j}(0)\right\}-1\right]+\sum_{0 \neq \alpha \in T} \sum_{i=1} \sum_{j=1} \min \left\{p_{i}(\alpha), q_{j}(\alpha)\right\} \geq 0 .
$$

Since all terms are non-negative, we see that $\nu(G)=\nu(A) \cdot \nu(B)$ if and only if there are no common eigenvalues besides zero and for the zero eigenvalue

$$
\sum_{i=1} \sum_{j=1}\left[\min \left\{p_{i}(0), q_{j}(0)\right\}-1\right]=0
$$

That is, $\min \left(p_{i}, q_{j}\right)=1$ for all $i=1, \ldots, \nu(A), j=1, \ldots, \nu(B)$. Hence if some $p_{i}(0)>1$ then all $q_{j}(0)>1$ or if some $q_{j}(0)=1$ then all $p_{i}(0)=1$. That is, either all elementary divisors of $A$ associated with zero are linear or all those of $B$ are. Thus the product rule holds if and only if either $\psi_{B}(x)=x f(x)$ or $\psi_{B}(x)=x g(x)$, where $(x, f)=1=(x, g)$. In other words, the product rule holds if and only if $A$ and $B$ have at most the zero eigenvalue in common and either $A^{\#}$ or $B^{\#}$ or both, exist.

Next we consider

$$
n_{0}(G)-\nu(G)=\sum_{\alpha \in T} \sum_{i=1}^{k(\alpha)} \sum_{j=1}^{t(\alpha)}\left[p_{i} q_{j}-\min \left(p_{i}, q_{j}\right)\right] \geq 0 .
$$

It thus follows that $n_{0}(G)=\nu(G)$, i.e. $G^{\#}$ exists, if and only if for each common eigenvalue $\gamma, p_{i} q_{j}=\min \left(p_{i}, q_{j}\right) \geq 1$, for all $i=1, \ldots, k, j=1, \ldots, t$. Next we note that if $r, s \geq 1$, then

$$
r s=\min \{r, s\} \text { if and only if } r=s=1
$$


and conclude that $G^{\#}$ exists if and only if for each common eigenvalue $\alpha$, the elementary divisors are linear. In other words, if and only if $\gamma \in T \Rightarrow \psi_{A}(x)=(x-\gamma) f(x)$ and $\psi_{B}(x)=$ $(x-\gamma) g(x)$, where $\gamma$ is not a root of $f(x)$ or $g(x)$.

\section{Remarks}

(i) If $G^{\#}$ exists then $\gamma \in T$ implies $(A-\gamma I)^{\#}$ and $(B-\gamma I)^{\#}$ both exist, yet $A^{\#}$ and/or $B^{\#}$ may not exist. For example, if $A$ is invertible and $\psi_{B}=x^{2} f(x)$ where $\operatorname{gcd}\left(\Delta_{A}, f\right)=1$, then the condition for $G^{\#}$ to exist are satisfied, yet $B^{\#}$ does not exist.

On the other hand, if $A^{\#}$ and $B^{\#}$ both exist, then $G^{\#}$ need not exist since they could have common e-values other than zero.

(ii) We know that if $G^{\#}$ exists then it is a polynomial in $G$, the coefficients of which can be derived from $\Delta(G)$, which in turn can be found from the eigenvalues of $A$ and $B$. Since this becomes intractable, we shall proceed differently. First an alternative proof of the above which is based on the property of Jordan blocks.

(iii) Since $G^{T}$ is similar to $\left(A^{T} \otimes I-I \otimes B\right)$ and $\psi_{A}=\psi_{A^{T}}$ we may interchange the roles of $A$ and $B$ to deduce the desired symmetry of Theorem 2.1.

To compute $G^{\#}$ suppose that $\beta_{i} \notin \sigma(A)$, for $i=1, \ldots, t$, and $\beta_{i} \in \sigma(A)$, for $i=$ $t+1, \ldots, v$. Next let $Q=\left[Q_{1}, \cdots, Q_{v}\right]$ and $Y=\left(Q^{T}\right)^{-1}=\left[Y_{1}, \cdots, Y_{v}\right]$ so that $B Q_{i}=$ $Q_{i} J_{q_{i}}\left(\beta_{i}\right)$ and $B_{i}^{T}=Y_{i} J_{q_{i}}^{T}\left(\beta_{i}\right)$. Then

$$
\begin{aligned}
& G^{\#}=(Y \otimes I)\left[\begin{array}{ccc|ccc}
G_{1}^{-1} & & & & & 0 \\
& \ddots & & 0 & & \\
0 & & G_{t}^{-1} & & & \\
\hline 0 & & G_{t+1}^{\#} & & \\
& & & \ddots & \\
& & & & & G_{v}^{\#}
\end{array}\right]\left(Q^{T} \otimes I\right) \\
&=\sum_{i=1}^{t} Y_{i} G_{i}^{-1} Q_{i}^{T}+\sum_{i=t+1}^{v} Y_{i} G_{i}^{\#} Q_{i}^{T} . \\
&
\end{aligned}
$$

Now $G_{i}^{-1}$ is given as in $(2.1)$ in which $\left(A-\beta_{i} I\right)^{-r}$ can be calculated from the spectral theorem [3]. Indeed,

$$
\left(A-\beta_{i} I\right)^{-r}=\sum_{k=1}^{s} \sum_{j=0}^{m_{k}-1}\left[\left(x-\beta_{i}\right)^{-r}\right]_{\lambda_{k}}^{(j)} Z_{k}^{j}=\sum_{k=1}^{s} \sum_{j=0}^{m_{k}-1}(-1)^{j} \frac{(r+j-1) !}{(r-1) !}\left(\lambda_{k}-\beta_{i}\right)^{-r-j} Z_{k}^{j} .
$$


Furthermore $\left(A-\beta_{i} I\right)^{\#}=g(A)$ where $g(x)=\left\{\begin{array}{cc}0 & x=\beta_{i} \\ 1 /\left(x-\beta_{i}\right) & x \neq \beta_{i}\end{array}\right.$ and so

$$
\left(A-\beta_{i} I\right)^{\#}=\sum_{k=1}^{s} \sum_{j=0}^{m_{k}-1} g^{(j)}\left(\lambda_{k}\right) Z_{k}^{j}=\sum_{\lambda_{k} \neq \beta_{j}} \sum_{j=0}^{m_{k}-1} \frac{(-1)^{j}}{\left(\lambda_{k}-\beta_{i}\right)^{j+1}} Z_{k}^{j} .
$$

Substituting these in the above yields $G^{\#}$.

Let us now turn to the case of an arbitrary field.

\section{The Arbitrary Field Case}

We shall now give conditions for $G^{\#}$ to exist in term of the invariant factors $\left\{a_{1}(x), . ., a_{r}(x)\right\}$ of $A$, and $\left\{b_{1}(x), . ., b_{s}(x)\right\}$ of $B$, and compute $G^{\#}$ in terms of polynomial matrices associated with $A$ and/or $B$.

We begin by reducing $A$ and $B$ to their respective rational canonical forms and as such reduce the problem to one where we have two companion matrices [3, p. 163], i.e., $P^{-1} A P=A_{c}=\operatorname{diag}\left[L\left(a_{1}(x)\right), \ldots, L\left(a_{r}(x)\right)\right]$ and $Q^{-1} B Q=B_{c}=\operatorname{diag}\left[L\left(b_{1}(x), \ldots, L\left(b_{s}(x)\right]\right.\right.$.

The nivellateur becomes

$$
\left(Q^{T} \otimes P^{-1}\right) G\left(Q^{-T} \otimes P\right)=I_{n} \otimes A_{c}-B_{c}^{T} \otimes I_{m}
$$

We permute the diagonal blocks using the "universal flip" matrix - see [3] - to get

$$
G \approx \oplus_{i=1}^{r} \oplus_{j=1}^{s} G_{i j}
$$

where $G_{i j}=I_{n_{i}} \otimes L\left[a_{i}(x)\right]-L^{T}\left[b_{j}(x)\right] \otimes I_{m_{j}}$.

We now replace $G$ by $G_{i j}$ and consider the "two-companion" case where $\mathrm{G}=I_{n} \otimes L[a(x)]-$ $L^{T}[b(x)] \otimes I_{m}$, with $b(x)=b_{0}+b_{1} x+\cdots+b_{n} x^{n}$.

Following [3] we reduce $x I-L^{T}[b(x)]$ to its Smith Normal From via

$$
R(x)\left[x I-L^{T}(b)\right] K(x)=\left[\begin{array}{cc}
b(x) & 0 \\
0 & I_{n-1}
\end{array}\right]
$$

where $R(x)=\left[\begin{array}{cc}\boldsymbol{\beta}^{T}(x) & 1 \\ -I & 0\end{array}\right], K(x)$ is as in lemma $(2.1)$ and $\left[\boldsymbol{\beta}^{T}(x), 1\right]=\left[b_{0}(x), \ldots, b_{n-2}(x), 1\right]$. In this the $b_{i}(x)$ are the adjoint polynomials defined by $\left[\boldsymbol{\beta}^{T}(x), 1\right]=\left[b_{1}, \ldots, b_{n}\right] K(x)$. We recall in passing that $\operatorname{adj}(x I-B)=\sum_{i=0}^{n-1} b_{i}(B) x^{i}$. Solving this gives

$$
\left[x I-L^{T}(b)\right]=R(x)^{-1}\left[\begin{array}{cc}
b(x) & 0 \\
0 & I_{n-1}
\end{array}\right] K(x)^{-1},
$$


and subsequently replacing $x$ by $A=L[a(x)]$ throughout, these polynomial identities we arrive at

$$
G=R(A)^{-1}\left[\begin{array}{cc}
b(A) & 0 \\
0 & I_{n-1}
\end{array}\right] K(A)^{-1}=P D Q .
$$

Since $P$ and $Q$ are invertible we may use [10, Corollary 2], which says that $(P D Q)^{\#}$ exists if and only if $U=D Q P D D^{-}+I-D D^{-}$is invertible. Since

$$
(1-a b)^{-1}=1+a(1-b a)^{-1} b
$$

this is equivalent to $U^{\prime}=D Q P+I-D D^{-}$being invertible, i.e. to $W=D+(I-$ $\left.D D^{-}\right) R(A) K(A)$ being invertible.

Theorem 3.1. $W$ is invertible if and only if $G^{\#}$ exists.

To compute $R(x) K(x)$ we define $T(x)=\left[\begin{array}{cc}\mathbf{b}^{T} & 1 \\ -K_{n-1}^{-1} & 0\end{array}\right]$, where $\mathbf{b}^{T}=\left[b_{1}, \ldots, b_{n}\right]$. Then $T(x) K_{n}(x)=R(x)=\left[\begin{array}{cc}\boldsymbol{\beta}^{T}(x) & 1 \\ -I_{n-1} & 0\end{array}\right]$ and

$$
R(x) K(x)=T(x) K(x)^{2}=\left[\begin{array}{cc}
\mathbf{b}^{T} & 1 \\
-K_{n-1}^{-1} & 0
\end{array}\right]\left[\begin{array}{cc}
K_{n-1}^{2}(x) & 0 \\
? & 1
\end{array}\right]=\left[\begin{array}{cc}
\gamma^{T}(x) & 1 \\
-K_{n-1}(x) & \mathbf{0}
\end{array}\right]
$$

in which $\boldsymbol{\gamma}^{T}(x)=\left[b^{\prime}(x), \boldsymbol{\rho}^{T}(x)\right]$ and $\boldsymbol{\rho}^{T}=\left[b_{0}^{\prime}(x), \ldots, b_{n-3}^{\prime}(x)\right]$. These contain the formal derivatives of the adjoint polynomials.

We next form

$$
\begin{aligned}
\left(I-D D^{-}\right) R(A) K(A) & =\left[\begin{array}{cc}
I-b(A) b(A)^{-} & 0 \\
0 & 0
\end{array}\right]\left[\begin{array}{cc}
{\left[b^{\prime}(A), \boldsymbol{\rho}^{T}(A)\right]} & 1 \\
? & ?
\end{array}\right] \\
& =\left[\begin{array}{cc}
{\left[I-b(A) b(A)^{-}\right] b^{\prime}(A)} & C \\
0 & 0
\end{array}\right]
\end{aligned}
$$

where $C=\left[I-b(A) b(A)^{-}\right]\left[\boldsymbol{\rho}^{T}(A), I\right]$. Adding in $D=\left[\begin{array}{cc}b(A) & 0 \\ 0 & I_{n-1}\end{array}\right]$ we arrive at

$$
W=\left[\begin{array}{cc}
b(A)+\left[I-b(A) b(A)^{-}\right] b^{\prime}(A) & C \\
0 & I
\end{array}\right] \text {. }
$$

This will be invertible exactly when $b(A)+\left[I-b(A) b(A)^{-}\right] b^{\prime}(A)$ is invertible. Note that $b(A)$ and $b^{\prime}(A)$ commute, but that $b(A)^{-}$need not be a polynomial in $A$.

We now need 
Lemma 3.1. Suppose $R$ is a von Neumann finite regular ring and $a h=h a$.

If $a+\left(1-a a^{-}\right) h$ is a unit then $a^{\#}$ must exist.

Proof. Let $u=a+\left(1-a a^{-}\right) h$. Then $u a=a^{2}+\left(1-a a^{-}\right) h a=a^{2}+\left(1-a a^{-}\right) a h=a^{2}$ and thus $a=u^{-1} a^{2}$. Since $R$ is finite we may conclude that $a^{\#}$ exists.

Suppose now that $W$ is invertible. Then $b(A)$ is GP and we can replace $b(A)^{-}$by $b(A)^{\#}=g(A)$ in $W$, implying that

Theorem 3.2. $W$ is a unit if and only if $b(A)$ is GP and $f(A)=b(A)+\left[I-b(A) b(A)^{\#}\right] b^{\prime}(A)$ is a unit.

We shall now reduce these conditions to suitable polynomial results.

First we recall the trivial gcd result

Lemma 3.2. $(u, d)=1$ if and only if $(d m+u, d)=1$.

and the group inverse result

Lemma 3.3. Suppose $M$ has minimal polynomial $\psi_{M}(x)$, and let $f(x)$ be a polynomial with $d(x)=\operatorname{gcd}\left(f(x), \psi_{M}(x)\right)$. The following are equivalent:

(i) $f(M)^{\#}$ exists (ii) $d(M)^{\#}$ exists (iii) $(d, \psi / d)=1 \quad$ (iv) $(f, \psi / d)=1$.

The proof is left as an exercise.

The latter says that if $f=p^{r} \tilde{f}$ and $\psi=p^{s} \tilde{\psi}$ for some prime factor $p$, with $(\tilde{f}, p)=1=$ $(p, \tilde{\psi})$, then $r \geq s$. In other words, common factors of $f$ and $\psi$ occur with minimal degree in $\psi_{M}$.

Since we may interchange $L(a)$ and $L(b)$ we must actually have that $r=s$. In other words the common prime factors of any invariant factor $a(x)$ of $A$ and any invariant factor $b(x)$ of $B$ must have the same multiplicity.

Now recall that $\psi_{A}=a(x)$ and set $(a, b)=d$. Then $b=d \tilde{b}$ and $a=d \tilde{a}$ for some $\tilde{b}, \tilde{a}$, with $(\tilde{a}, \tilde{b})=1$. Moreover $b(A)$ has a group inverse if and only if $(d, \tilde{a})=1$ or if $(b, \tilde{a})=1$.

The existence of $b(A)^{\#}$ also says that $b(A)^{2} g(A)=b(A)$ which holds iff $a \mid b(1-b g)$ iff $d \tilde{a} \mid d \tilde{b}(1-g b)$ iff $\tilde{a} \mid \tilde{b}(1-g b)$. But $(\tilde{a}, \tilde{b})=1$ and thus $\tilde{a} \mid(1-g b)$ and conversely. We may as such write $1-g b=\tilde{a} h$, for some $h(x)$. This ensures that $(\tilde{a}, b)=1=(\tilde{a}, g)$ and gives $f=b+\tilde{a} h b^{\prime}$.

Next recall, by Hensel's theorem [8, p. 21, Theorem 15.5], that $f(A)$ is invertible if and only if $(f, a)=1$, i.e. if and only if $(f, d)=1=(f, \tilde{a})$. First we observe that $(f, d)=1$ if 
and only if $\left(b+(1-b g) b^{\prime}, d\right)=1$ if and only if $\left(d \tilde{b}\left(1-g b^{\prime}\right)+b^{\prime}, d\right)=1$. By Lemma (3.2) this happens precisely when $\left(b^{\prime}, d\right)=1$.

Next we note that because $b=d \tilde{b}$ we have $b^{\prime}=d^{\prime} \tilde{b}+d(\tilde{b})^{\prime}$ and thus again by the lemma, $\left(b^{\prime}, d\right)=1$ if and only if $\left(d^{\prime} \tilde{b}+d(\tilde{b})^{\prime}, d\right)=1$ if and only if $\left(d^{\prime} \tilde{b}, d\right)=1$ if and only if $\left(d, d^{\prime}\right)=1=(\tilde{b}, d)=1$.

Since $(\tilde{a}, \tilde{b})=1$ it follows that $(a, \tilde{b})=(d \tilde{a}, \tilde{b})=1$ so that $\tilde{b}(A)$ is invertible.

We now cancel $\tilde{b}(A)$ in $d(A)^{2} \tilde{b}(A)^{2} g(A)=b(A)^{2} g(A)=b(A)=d(A) \tilde{b}(A)$. This implies that

$$
d(A)^{2} \tilde{b}(A) g(A)=d(A)
$$

so that $d(A)^{\#}$ exists and

$$
d(A)^{\#}=g(A) \tilde{b}(A) \text { and } b(A) b(A)^{\#}=d(A) d(A)^{\#} .
$$

The surprising fact is that the condition (f, $\tilde{a})=1$ automatically follows if $b(A)$ is GP.

Indeed, we have

$$
b(a)^{\#} \text { exists } \Rightarrow(b, \tilde{a})=1 \Rightarrow\left(b+\tilde{a} h b^{\prime}, \tilde{a}\right)=1 \Rightarrow\left(b+(1-b g) b^{\prime}, \tilde{a}\right)=1 \Rightarrow(f, \tilde{a})=1 .
$$

We recap in

Theorem 3.3. If $G=I_{n} \otimes L[a(x)]-L^{T}[b(x)] \otimes I_{m}$, then $G^{\#}$ exists if and only if $(d, \tilde{a})=$ $1=\left(d, d^{\prime}\right)$, where $d=(a, b)$ and $a=d \tilde{a}$.

Now $\left(d, d^{\prime}\right)=1$ means that $d$ only has simple prime factors. As a consequence, the common invariant factors have simple prime factors. For the closed field case, this says that all elementary divisors corresponding to common eigenvalues must be linear - as we met in the previous section.

To compute the actual inverse of $f(A)$ we observe that because $\left(d, d^{\prime}\right)=1$, we can find $s$ and $t$ by Euclid's algorithm, such that $d(x) s(x)+d^{\prime}(x) t(x)=1$. This means that

$$
d^{\prime}(A) t(A)=1-d(A) s(A) .
$$

Substituting for $b^{\prime}$ we may rewrite $f(A)=b(A)+[I-b(A) g(A)] b^{\prime}(A)$ as $f(A)=$ $b(A)+\left[I-d(A) d(A)^{\#}\right] d^{\prime}(A) \tilde{b}(A)$, which we may invert to give

$$
f(A)^{-1}=b(A)^{\#}+\left[I-d(A) d(A)^{\#}\right] \tilde{b}(A)^{-1} t(A) .
$$

Indeed, this follows because

$$
\begin{aligned}
{\left[I-d(A) d(A)^{\#}\right] d^{\prime}(A) \tilde{b}(A) \cdot \tilde{b}(A) t(A) } & =\left[I-d(A) d(A)^{\#}\right] d^{\prime}(A) t(A) \\
& =\left[I-d(A) d(A)^{\#}\right][I-d(A) s(A)] \\
& =I-d(A) d(A)^{\#}
\end{aligned}
$$


Remark We could have used the fact that $\left(b^{\prime}, d\right)=1$ which gives $b^{\prime} u=1-d v$ for some $v(x)$ and write $f(A)^{-1}=b(A)^{\#}+\left[I-b(A) b(A)^{\#}\right] u(A)$. The computation of $u$, however, is more difficult than that of $t(x)$.

Since $d(x)$ only has simple pime factors, the computation of $t(A)$ can be done via the gcd algorithm and the Chinese remainder theorem. Indeed, suppose $d=p_{1} p_{2} \cdots p_{k}$, where the $p_{i}$ are distinct prime polynomials. Further set $M_{i}=\frac{d}{p_{i}}$ and $g_{i}=M_{i}^{-1} \bmod p_{i}$. Next we observe that if $s d+t d^{\prime}=1$, then $t=\left(d^{\prime}\right)^{-1} \bmod d$, which is equivalent to $t=\left(d^{\prime}\right)^{-1} \bmod p_{i}$ for all $i=1, \ldots, k$. Because $d^{\prime}=p_{1}^{\prime} M_{1}+p_{2}^{\prime} M_{2}+\ldots$ we see that $\left(d^{\prime}\right)^{-1}$ $\bmod p_{i}=\left(p_{i}^{\prime} M_{i}\right)^{-1} \bmod p_{i}=g_{i}\left(p_{i}^{\prime}\right)^{-1} \bmod p_{i}$. Using the Chinese remainder theorem we may conclude that

$$
t=\sum_{i=1}^{k} g_{i}^{2} M_{i}\left(p_{i}^{\prime}\right)^{-1} \bmod p_{i}
$$

\section{Computation of $G^{\#}$}

We may compute the actual group inverse of $G$ via the formula [10],

$$
\begin{aligned}
G^{\#} & =P U^{-2} D Q=R(A)^{-1}\left[I+\left(I-D K(A)^{-1} R(A)^{-1}\right)\left(U^{\prime}\right)^{-1} D D^{-}\right]^{2} D K(A)^{-1} \\
& =R(A)^{-1}\left[I+(R K-D) W^{-1} D D^{-}\right]^{2} D K(A)^{-1},
\end{aligned}
$$

in which $\left(U^{\prime}\right)^{-1}=P^{-1} Q^{-1} W^{-1}=R(A) K(A) W^{-1}$ and $W^{-1}=\left[\begin{array}{cc}f(A)^{-1} & -f(A)^{-1} C \\ 0 & I\end{array}\right]$.

First we see that

$$
W^{-1} D D^{-}=\left[\begin{array}{cc}
f(A)^{-1} b(A) b(A)^{\#} & -f(A)^{-1} C \\
0 & I
\end{array}\right] .
$$


Hence

$$
\begin{gathered}
R(A) K(A) W^{-1} D D^{-}=\left[\begin{array}{c|cc}
b^{\prime}(A) & \boldsymbol{\rho}^{T}(A) & I \\
\hline-I & 0 & 0 \\
-\left[\begin{array}{c}
A \\
A^{2} \\
\vdots \\
A^{n-2}
\end{array}\right] & \\
-K_{n-2}(A) & 0
\end{array}\right]\left[\begin{array}{cc}
f(A)^{-1} b(A) b(A)^{\#} & -f(A)^{-1} C \\
0 & I
\end{array}\right] \\
=\left[\begin{array}{c|c}
b^{\prime}(A) f(A)^{-1} b(A) b(A)^{\#} \\
-\left[\begin{array}{c}
I \\
A \\
\vdots \\
A^{n-2}
\end{array}\right] f(A)^{-1} b(A) b(A)^{\#} \\
-\left[\begin{array}{c}
I \\
A \\
\vdots \\
A^{n-2}
\end{array}\right] f(A)^{-1} C+\left[\begin{array}{cc}
0 \\
-K_{n-2}(A) & 0
\end{array}\right]
\end{array}\right] .
\end{gathered}
$$

Recalling the definition of $C$ we see that the $(1,2)$ entry becomes

$$
\boldsymbol{\sigma}^{T}=\left[I-b^{\prime}(A) f(A)^{-1}\left(I-b(A) b(A)^{\#}\right)\right]\left[\boldsymbol{\rho}(A)^{T}, I\right] .
$$

On the other hand,

$$
D W^{-1} D D^{-}=\left[\begin{array}{cc}
b(A) f(A)^{-1} b(A) b(A)^{\#} & f(A)^{-1} b(A) C \\
0 & I
\end{array}\right]=\left[\begin{array}{cc}
f(A)^{-1} b(A) & 0 \\
0 & I
\end{array}\right],
$$

because $b(A) C=0$.

Whence $U^{-1}=I+(R K-D) W^{-1} D D^{-}$takes the form

$U^{-1}=\left[\begin{array}{c}I+f(A)^{-1} b(A)\left[b^{\prime}(A) b(A)^{\#}-I\right] \\ -\left[\begin{array}{c}I \\ A \\ \vdots \\ A^{n-2}\end{array}\right] f(A)^{-1} b(A) b(A)^{\#} \mid I-\left[\begin{array}{c}I \\ A \\ \vdots \\ A^{n-2}\end{array}\right] f(A)^{-1} C+\left[\begin{array}{cc}0 & \sigma^{T}(A) \\ -K_{n-2}(A) & 0\end{array}\right]\end{array}\right]$

This we substitute in

$G^{\#}=R(A)^{-1}\left[I+(R(A) K(A)-D) W^{-1} D D^{-}\right]\left[I+(R(A) K(A)-D) W^{-1} D D^{-}\right] D K(A)^{-1}$,

which is not conducive to simplification. 


\section{Open Questions and remarks}

We end with some pertinent questions and remarks.

1. Squaring the matrix $U^{-1}$ does not look appealing!

2. The expression for $G^{\#}$ should be "symmetric" in $L(a)$ and $L(b)$, i.e $a(x)-b(x)$ symmetric, and as such there should be some simplification.

3. Can we find a good representation for $\left(p^{\prime}\right)^{-1} \bmod p$ for a prime polynomial $p(x)$ ?

4. Can we find the polynomial $g(A)=A^{\#}$ ?

5. Can Lemma (3.1) be extended to regular rings?

6. Can we use the invertibility of $a g+1-a a^{-}$to get a better result?

\section{Acknowledgment}

The authors thank an anonymous referee for his/her valuable corrections.

\section{References}

[1] A. Ben-Israel and T.N.E.Greville, Generalized Inverses: Theory and Applications, Wiley, New York, 1974.

[2] R.E. Hartwig, The resultant and the matrix equation $A X=X B$. SIAM J. Appl. Math. 22 (1972), 538-544.

[3] R.E. Hartwig, $A X-X B=C$, resultants and generalized inverses. SIAM J. Appl. Math. 28 (1975), 154-183.

[4] R.E. Hartwig, Applications of the Wrońskian and Gram matrices of $\left\{t^{i} e^{k}\right\}$. Linear Algebra Appl. 43 (1982), 229-241.

[5] V. Kucera, The matrix equation $A X+X B=C$. SIAM J. Appl. Math. 26 (1974), $15-25$.

[6] P. Lancaster and M. Tismenetsky, The Theory of Matrices: With Applications, 2nd Edition, Academic Press, 1985. 
[7] V. Lovass-Nagy and D.L. Powers, A note on block diagonalization of some partitioned matrices. Linear Algebra and Appl. 5 (1972), 339-346.

[8] C. C. MacDuffee, The Theory of Matrices, Chelsea, New York, 1956.

[9] P. Patricio and R.E. Hartwig, The link between regularity and strong-pi-regularity. $J$. Aust. Math. Soc. 89 (2010), no. 1, 17-22.

[10] R. Puystjens and R.E. Hartwig, The group inverse of a companion matrix. Linear and Multilinear Algebra 43 (1997), no. 1-3, 137-150. 